\section{Knochenmarkläsionen und orale Bisphosphonate bei Arthrose}

Ballal $P$ et al. The relation of oral bisphosphona-

tes to bone marrow lesion volume among

women with osteoarthritis. Osteoarthritis

Cartilage 2020; 28: 1325-1329

Arthrose betrifft das ganze Gelenk, inklusive der Knochenstruktur. Eine Folge können Knochenmarkläsionen sein, welche Fibrosierungen, Nekrosen und Ödeme aufweisen. Die Veränderungen sind auf MRT-Aufnahmen sichtbar und verschlechtern die Diagnose. Forscher setzen Hoffnung auf Bisphosphonate zur Verbesserung der Knochenmarkläsionen. Da bisherige Studien zu unterschiedlichen Ergebnissen kommen, haben Ballal et al. diese Kohortenstudie durchgeführt.

Die Studienteilnehmer wurden durch die Osteoarthritis Initiative, eine Längsschnittkohorte von Menschen mit oder ohne Risiko für Knie-Arthrose ( $n=4796$, Alter 4579), rekrutiert. Einschlusskriterium war das weibliche Geschlecht, da Bisphosphonate hauptsächlich Frauen verschrieben werden. Ausschlusskriterium war die gleichzeitige Einnahme weiterer Medikamente, welche auf den Knochen wirken. Außerdem wurde nur eingeschlossen, wenn sowohl eine MRT-Aufnahme zu Beginn als auch nach 12 Monaten vorlag. Die Einnahme von Bisphosphonaten erfolgte durch Selbstangabe der Patientinnen. Frauen ohne Einnahme wurden in die Vergleichsgruppe aufgenommen. Als Ergebnisparameter fungiert das Ausmaß der Knochenmarkläsion. Dieses wurde auf MRT-Aufnahmen ausgemessen, das Vorgehen ist in der Originalstudie beschrieben. Die Auswertung fand anhand von Propensity Score Matching statt. In das Model wurden verschiedene Parameter aufgenommen, wie z. B. Alter, BMI, Rauchstatus, Einkommen, diverse Erkrankungen, Ernährung, Schweregrad der Arthrose und Schmerz. Es wurde die Veränderung der Knochenmarkläsion innerhalb von $12 \mathrm{Mo}$ naten untersucht und zwischen Frauen mit Bisphosphonat-Einnahme und ohne verglichen.
Von den Frauen wurde die Einnahme folgender Bisphosphonate berichtet: Alendronat, Risedronat und Ibandronat. Insgesamt konnten 145 Frauen mit Bisphosphonat-Einnahme eingeschlossen werden. Dazu wurden 145 Frauen ohne Einnahme gematcht. Das Durchschnittsalter lag bei 65 Jahren und der BMI bei 26. Das Auftreten von Knochenmarkläsionen zu Beginn war in beiden Gruppen ähnlich (51\% Bisphosphonat, $47 \%$ Kontrollgruppe). Die Veränderung des Knochenmarkläsions-Volumens, unabhängig vom Auftreten zu Beginn, zeigte zwischen den Gruppen keinen signifikanten Unterschied. Betrachtet man nur die Patientinnen mit Läsion zu Beginn, so zeigt sich, dass die Bisphosphonateinnahme eher dazu beiträgt das Volumen zu verringern als ohne Einnahme ( $48 \%$ zu $41 \%$ ).

\section{FAZIT}

Knochenmarkläsionen sind eine Folge von Arthrose und gehen häufig mit Schmerzen einher. In der Forschung wird der Einsatz von Bisphosphonaten zur Verringerung des Läsionsvolumens diskutiert. Die Autoren konnten keinen signifikanten Effekt feststellen. Dies deckt sich mit der bisherigen unklaren Studienlage. Sie merken jedoch an, dass über den Zeitraum eines Jahres keine Nebenwirkungen festgestellt werden konnten. Dies bietet zumindest die Chance eine Therapie mit Bisphosphonaten zu versuchen.

Stephanie Burgess, Stuttgart 